\title{
Asymmetric Michael Addition of Ketones to Nitroolefins Catalyzed by a New Chiral Catalyst
}

\author{
Lian-Jun Wang* and Feng-Feng Hu
}

\author{
College of Chemistry and Chemical Engineering, Hunan Institute of Engineering, Hunan 411104, P. R. China \\ *E-mail: wangljchem@gmail.com \\ Received February 11, 2010, Accepted March 13, 2010
}

\begin{abstract}
A new chiral catalyst was synthesized and found that it could catalyzed the asymmetric Michael reaction of ketones with nitroolefins smoothly at room temperature, giving the desired adducts in $71-92 \%$ yields with excellent diastereoselectivities and high enantioselectivities (up to $95 \%$ ee).
\end{abstract}

Key Words: Chiral catalyst, Michael reaction, Nitroolefin, Ketone, Enantioselectivity

\section{Introduction}

Chiral asymmetric Michael addition of nucleophiles to nitroolefins affords very useful chiral building blocks in organic synthesis. ${ }^{1}$ Many outstanding efforts have been taken into the development of both chiral metal-catalysts ${ }^{1,2}$ and chiral organocatalysts $^{1,3}$ for the nitro-Michael addition. Due to the importance of the nitro-Michael addition and nitro group as a "synthetic chameleon", the development of new chiral catalysts for a highly enantioselective nitro-Michael reaction is still significant. Herein, we wish to report a new chiral multifunctional catalyst for the highly stereoselective Michael addition of nitroolefins with ketones.

As we know, chiral thiourea organocatalysts 1 were applied successfully in the asymmetric Michael reaction of nitroolefins with malonates or ketones. ${ }^{4,5,10}$ Considering that the hydrogen connecting to nitrogen in thiourea may play a key role in the asymmetric nitro-Michael reaction, ${ }^{4 \mathrm{c}-\mathrm{e}}$ we envision to synthesize new catalyst in order to increase its activity as a Brønsted acid catalyst.

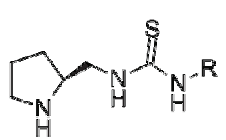

1

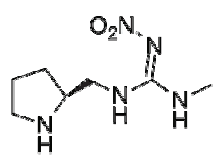

2

\section{Results and Discussion}

Aminomethylpyrrolidine 3 can be prepared from L-proline according to the literature method. ${ }^{6}$ It was found that the reaction of 3 with $\mathbf{4}^{7 \mathrm{a}}$ in $\mathrm{CH}_{2} \mathrm{Cl}_{2}$ at room temperature for $5 \mathrm{hr}$, followed by stirring the resulting mixture with $\mathrm{CH}_{3} \mathrm{NH}_{2}$ at this temperature for $1 \mathrm{hr}$, then deprotected by trifluoroacetic acid to afford desired catalyst 2 in $72 \%$ yield (Scheme 1). ${ }^{7 \mathrm{~b}}$

Initially, chiral thiourea organocatalysts $\mathbf{2}$ were examined in the Michael reaction of cyclohexanone 5a with trans-nitrostyrene 6a. As Table 1 shows, using $10 \mathrm{~mol} \% \mathbf{2}$ in the common solvents such as $\mathrm{MeOH}$, THF, $\mathrm{CH}_{2} \mathrm{Cl}_{2}$, Toluene, EtOH, $\mathrm{CH}_{3} \mathrm{CN}$ and $\mathrm{H}_{2} \mathrm{O}$ led to the adduct 7a in low yields (entries 1-8). Our experiment showed that adducts 7a was obtained in moderate yields when DMF or DMSO was used as solvent (entries 9 and 10). Interestingly, using dichloromethane (DCE) as solvent improved both the yield and the enantioselectivity (compare

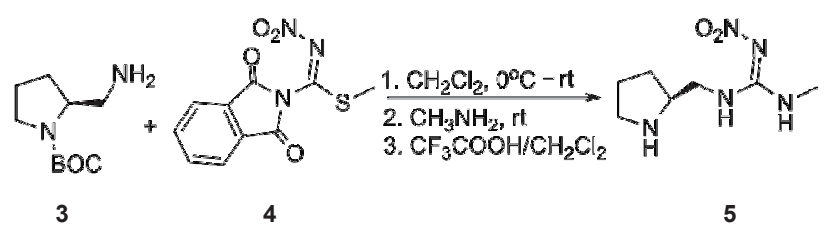

Scheme 1. Synthesis of chiral catalyst 2

Table 1. Optimization of the Michael reaction of cyclohexanone with trans-nitrostyrene ${ }^{2 \mathrm{a}}$

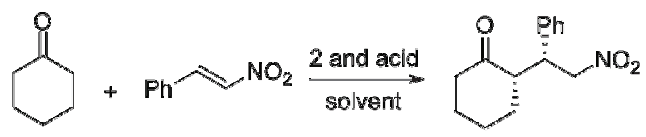

$5 a \quad 6 a \quad 7 a$

\begin{tabular}{ccccccc}
\hline Entry & Solvent & Additive & $\begin{array}{c}\text { Time } \\
(\mathrm{h})\end{array}$ & $\begin{array}{c}\text { Yield } \\
(\%)\end{array}$ & d.r. $^{b}$ & $\begin{array}{c}\text { e.e. }^{c} \\
(\%)\end{array}$ \\
\hline 1 & $\mathrm{MeOH}$ & - & 36 & 11 & n.d. & n.d. \\
2 & $\mathrm{THF}$ & - & 36 & 16 & n.d. & n.d. \\
3 & $\mathrm{CH}_{2} \mathrm{Cl}_{2}$ & - & 36 & 22 & n.d. & n.d. \\
4 & $\mathrm{Toluene}_{5}$ & - & 36 & 9 & n.d. & n.d. \\
5 & $\mathrm{CHCl}_{3}$ & - & 36 & 31 & n.d. & n.d. \\
6 & EtOH & - & 36 & 21 & n.d. & n.d. \\
7 & $\mathrm{CH}_{3} \mathrm{CN}$ & - & 36 & 33 & n.d. & n.d. \\
8 & $\mathrm{H}_{2} \mathrm{O}$ & - & 36 & 8 & n.d. & n.d. \\
9 & $\mathrm{DMF}$ & - & 36 & 56 & $75 / 25$ & 68 \\
10 & $\mathrm{DMSO}$ & - & 36 & 68 & $80 / 20$ & 74 \\
11 & $\mathrm{DCE}$ & - & 24 & 80 & $85 / 15$ & 80 \\
12 & $\mathrm{DCE}$ & $\mathrm{TFA}_{12}$ & 24 & 87 & $88 / 12$ & 84 \\
13 & $\mathrm{DCE}$ & $\mathrm{HCO}_{2} \mathrm{H}$ & 24 & 86 & $90 / 10$ & 86 \\
14 & $\mathrm{DCE}$ & $\mathrm{CH}_{3} \mathrm{CO}_{2} \mathrm{H}$ & 24 & 87 & $92 / 8$ & 90 \\
15 & $\mathrm{DCE}$ & $\mathrm{PhCO}_{2} \mathrm{H}$ & 24 & 92 & $97 / 3$ & 95 \\
\hline
\end{tabular}

${ }^{a}$ Unless otherwise noted, all reactions were carried out in solvent ( $\left.1 \mathrm{~mL}\right)$ using $5 \mathbf{a}(0.14 \mathrm{~mL}, 5$ equiv. $)$ and $\mathbf{6 a}(0.25 \mathrm{mmol}, 1$ equiv. $)$ in the present of $10 \mathrm{~mol} \% 2$ at rt. ${ }^{b}$ Determined by ${ }^{1} \mathrm{H}$ NMR. ${ }^{c}$ Determined by chiral HPLC analysis (chiralpak AS-H). 
entries 1-10 with entry 11). Moreover, under the catalysis of 2 and in DCE solvent conditions, the addition of a catalytic amount of TFA resulted in excellent yield and enantiomeric excess (entry 12-15). When $\mathrm{HCO}_{2} \mathrm{H}$ or $\mathrm{CH}_{3} \mathrm{CO}_{2} \mathrm{H}$ was employed instead of TFA, the little higher yield and enantioselectivity of 7a was obtained (compare entry 13, 14 with 12). However, the addition of $\mathrm{PhCO}_{2} \mathrm{H}$ led to the remarkable improvement of yield and enantioselectivity (compare entry 15 with $12-14$ ). Thus, the optimal reaction conditions were as follows: the reaction was performed at room temperature with DCE as solvent, $10 \mathrm{~mol}$ $\%$ benzoic acid was employed as an additive and the amount of chiral catalyst 2 was $10 \mathrm{~mol} \%$.

Under the optimal conditions, the Michael reactions of nitroolefins with various ketones were examined (Table 2). It was found that chiral catalyst 2 at $10 \mathrm{~mol} \%$ loading could catalyze the Michael reaction of trans-nitroolefins $\mathbf{6 a - f}$ smoothly with cyclohexanone 5a at room temperature for 20 - $36 \mathrm{~h}$, giving the adducts $7 \mathbf{a}-\mathbf{f}$ in moderate to excellent yields (71 - 92\%) with excellent diastereoselectivities. The experimental results also demonstrated that for almost all ketones 5a-e, the chiral catalyst 2 could result in high enantioseletivities ( $85-94 \%$ ee) in the nitro-Michael reaction. The absolute configurations of the adducts 7a-j were determined by comparison with known literature data. ${ }^{3 \mathrm{~d}, 10}$ It can be concluded that chiral catalyst $\mathbf{2}$ is the same as chiral thiourea catalyst $\mathbf{1}$ which resulted in good yields and excellent enantioselectivities. This can be rationalized as follows: the hydrogens connecting to nitrogen in this catalyst may also coordinate one or two oxygens of nitro group. ${ }^{4 c-e}$

\section{Experiments}

${ }^{1} \mathrm{H}$ NMR and ${ }^{13} \mathrm{C}$ NMR spectra were determined in $\mathrm{CDCl}_{3}$ or $\mathrm{CD}_{3} \mathrm{OD}$ on a Brucker ARX-300 (300 MHz) with TMS as internal standard. Mass spectra were recorded by the ESI method with ThermoFisher Scientific LCQ FLEET mass spectrometer. Melting points $(\mathrm{mp})$ were determined with a digital electrothermal apparatus without further correction. Optical rotations were recorded on a Perkin Elemer 241 Polarimeter. The enantiomeric excesses of Michael adducts 6a-i were determined by chiral HPLC analysis on Waters 600 under the conditions as follows: Chiralpak AS-H column, hexane $/ i$-PrOH $=90 / 10, \lambda=$ $238 \mathrm{~nm}$, flow $=0.7 \mathrm{~mL} / \mathrm{min}$. Nitroolefins were prepared according to the literature procedures. ${ }^{8}$

Preparation of catalyst 2. To a solution of S-methyl- $N$ nitro- $N$-phthaloylisothiourea $(4,3.00 \mathrm{~g}, 11.3 \mathrm{mmol})$ in dichloromethane $(20 \mathrm{~mL})$ in an ice-cold bath, Aminomethylpyrrolidine $(3,2.26 \mathrm{~g}, 11.3 \mathrm{mmol})$ in dichloromethane $(10 \mathrm{~mL})$ was added dropwise. The mixture was stirred at room temperature for $3 \mathrm{hr}$. The resulting solid was filtered off and the filtrate was concentrated under reduced pressure.

The above crude product $(2.00 \mathrm{~g}, 6.45 \mathrm{mmol})$ and $40 \%$ $\mathrm{MeNH}_{2}$ (in $\mathrm{MeOH}, 1.00 \mathrm{~g}, 12.9 \mathrm{mmol}$ ) in $\mathrm{MeOH}(10 \mathrm{~mL})$ was stirred at room temperature for $1 \mathrm{hr}$. The mixture was concentrated to dryness under reduced pressure and the solid was washed with ether $(15 \mathrm{~mL})$, and then dried to give BOC protected product as a white solid, which was dissolved in the mixture of trifluoroacetic acid and dichloromethane $(10 \mathrm{~mL}, \mathrm{v} / \mathrm{v}=1: 2)$ and the solution was stirred at room temperature for $3 \mathrm{~h}$. Re-
Table 2. Michael reactions of trans-nitroolefins with ketones catalyzed by $\mathbf{2}^{a}$

\begin{tabular}{|c|c|c|c|c|c|c|c|}
\hline & $5 a$ & $6 a$ & & & $7 a$ & & \\
\hline 7 & $\mathrm{Ar}$ & $\mathrm{R}_{1}$ & $\mathrm{R}_{2}$ & $\begin{array}{l}\text { Time } \\
\text { (h) }\end{array}$ & $\begin{array}{c}\text { Yield } \\
(\%)\end{array}$ & d.r. ${ }^{b}$ & $\begin{array}{l}\text { ee } \\
(\%)^{c}\end{array}$ \\
\hline $\mathbf{a}$ & $\mathrm{Ph}$ & $\left(\mathrm{CH}_{2}\right)_{3}$ & - & 24 & 92 & $97 / 3$ & 95 \\
\hline b & $4-\mathrm{ClC}_{6} \mathrm{H}_{4}$ & $\left(\mathrm{CH}_{2}\right)_{3}$ & - & 28 & 86 & $90 / 10$ & 90 \\
\hline c & $4-\mathrm{MeOC}_{6} \mathrm{H}_{4}$ & $\left(\mathrm{CH}_{2}\right)_{3}$ & - & 22 & 83 & $96 / 4$ & 93 \\
\hline d & $4-\mathrm{BrC}_{6} \mathrm{H}_{4}$ & $\left(\mathrm{CH}_{2}\right)_{3}$ & - & 32 & 80 & $93 / 7$ & 94 \\
\hline e & 2-Furyl & $\left(\mathrm{CH}_{2}\right)_{3}$ & - & 20 & 88 & $95 / 5$ & 90 \\
\hline $\mathbf{f}$ & $2-\mathrm{BrC}_{6} \mathrm{H}_{4}$ & $\left(\mathrm{CH}_{2}\right)_{3}$ & - & 24 & 83 & $91 / 9$ & 91 \\
\hline g & $\mathrm{Ph}$ & $\mathrm{CH}_{3}$ & $\mathrm{CH}_{3}$ & 32 & 77 & $97 / 3$ & 92 \\
\hline h & $\mathrm{Ph}$ & $\mathrm{H}$ & $\mathrm{H}$ & 36 & 79 & - & 86 \\
\hline i & $\mathrm{Ph}_{4}$ & $\left(\mathrm{CH}_{2}\right)_{2}$ & - & 36 & 71 & - & 88 \\
\hline $\mathbf{j}$ & $\mathrm{Ph}$ & $\mathrm{CH}_{3}$ & $\mathrm{H}$ & 36 & 72 & - & 85 \\
\hline
\end{tabular}

${ }^{\bar{a}}$ All reactions were carried out in toluene ( $\left.1 \mathrm{~mL}\right)$ using 4 ( $0.14 \mathrm{~mL}, 5$ eqiv. $)$ and $5(0.25 \mathrm{mmol}, 1$ equiv. $)$ in the present of $10 \mathrm{~mol} \% 2$ and $10 \mathrm{~mol} \%$ $\mathrm{PhCO}_{2} \mathrm{H}$ at rt. ${ }^{b}$ Determined by ${ }^{1} \mathrm{H}$ NMR. ${ }^{c}$ Determined by chiral HPLC analysis (chiralpak AS-H).

moval of solvent gave a residue, which was washed with $\mathrm{Et}_{2} \mathrm{O}$ $(20 \mathrm{~mL})$, and then filtration led to a solid. To the mixture of the solid in $\mathrm{CHCl}_{3}$ was added saturated aqueous $\mathrm{NaHCO}_{3}$ to weak basicity. The organic layer was washed with saturated brine $(10 \mathrm{~mL} \times 3)$ and dried over $\mathrm{MgSO}_{4}$. Concentration and purification by column chromatography $\left(\mathrm{MeOH} / \mathrm{CH}_{2} \mathrm{Cl}_{2}\right)$ gave catalyst 2 .

White solid; mp 87 - $90{ }^{\circ} \mathrm{C}$; Yield: 72\%. ${ }^{1} \mathrm{H}$ NMR (300 MHz, $\left.\mathrm{CD}_{3} \mathrm{OD}\right) \delta 3.72(\mathrm{~s}, 3 \mathrm{H}), 3.37-3.29(\mathrm{~m}, 2 \mathrm{H}), 2.81(\mathrm{~s}, 3 \mathrm{H}), 2.27-$ $2.18(\mathrm{~m}, 2 \mathrm{H}), 2.16-1.95(\mathrm{~m}, 3 \mathrm{H}), 1.89-1.74(\mathrm{~m}, 2 \mathrm{H}) .{ }^{13} \mathrm{C} \mathrm{NMR}$ (75 MHz, CD $\left.{ }_{3} \mathrm{OD}\right) \delta 164.6,59.7,51.5,41.6,29.2,24.7,22.5$. MS (ESI, positive) $m / z 202.5(\mathrm{M}+\mathrm{H})$. Anal. Cald. for $\mathrm{C}_{7} \mathrm{H}_{15} \mathrm{~N}_{5} \mathrm{O}_{2}$ : C, 41.78; H, 7.51; N, 34.80. Found: C, 42.01; H, 7.24; N, 34.18.

Michael reaction of ketones 4 with trans-nitroolefins 5: $\mathrm{Ge}-$ neral procedure; The mixture of catalyst $2(6.0 \mathrm{mg}, 0.03 \mathrm{mmol})$, benzoic acid (3.6 mg, $0.03 \mathrm{mmol}$ ) and ketone 5 (4.5 mmol) was stirred at room temperature for $5 \mathrm{~min}$, and then nitroolefin 6 $(0.3 \mathrm{mmol})$ was added. After the reaction was completed (monitored by TLC), the mixture was concentrated under reduced pressure, and the resulting residue was purified by column chromatography (petroleum ether/AcOEt as eluent) to give the adduct 7 .

(S)-2-[(R)-2-Nitro-1-phenylethyl]cyclohexanone (6a): ${ }^{9}$ Yield: $92 \% ;[\alpha]_{\mathrm{D}}^{25}=-18.2\left(c=0.63\right.$ in $\left.\mathrm{CHCl}_{3}\right)$; HPLC analysis: $t_{\mathrm{R}}$ (minor) $=19.58 \mathrm{~min}, t_{\mathrm{R}}$ (major) $=30.62 \mathrm{~min}, 95 \%$ ee. ${ }^{1} \mathrm{HNMR}$ $\left(300 \mathrm{MHz}, \mathrm{CDCl}_{3}\right) \delta$ 7.41-7.20 (m, 3H), 7.18-7.10 (m, 2H), $4.92(\mathrm{dd}, J=4.7,12.6 \mathrm{~Hz}, 1 \mathrm{H}), 4.64(\mathrm{dd}, J=9.9,12.3 \mathrm{~Hz}, 1 \mathrm{H})$, $3.74(\mathrm{dt}, J=4.5,9.9 \mathrm{~Hz}, 1 \mathrm{H}), 2.70-2.60(\mathrm{~m}, 1 \mathrm{H}), 2.50-2.29$ (m, 2H), 2.15-2.01 (m, 1H), 1.80-1.45 (m, 4H), 1.31-1.20 (m, 1H).

(S)-2-((R)-1-(4-Chlorophenyl)-2-nitroethyl)cyclohexanone (6b): ${ }^{10}$ Yield: $86 \%$; $[\alpha]_{\mathrm{D}}^{25}=-19.7\left(c=0.40\right.$ in $\left.\mathrm{CHCl}_{3}\right)$; HPLC analysis: $t_{\mathrm{R}}($ minor $)=43.61 \mathrm{~min}, t_{\mathrm{R}}$ (major) $=56.54 \mathrm{~min}, 90 \%$ ee. ${ }^{1} \mathrm{H}$ NMR $\left(300 \mathrm{MHz}, \mathrm{CDCl}_{3}\right) \delta 7.33(\mathrm{~d}, J=8.4 \mathrm{~Hz}, 2 \mathrm{H}), 7.15$ (d, $J=8.4 \mathrm{~Hz}, 2 \mathrm{H}), 4.98$ (dd, $J=4.8,12.8 \mathrm{~Hz}, 1 \mathrm{H}), 4.65$ (dd, 
$J=10.0,12.8 \mathrm{~Hz}, 1 \mathrm{H}), 3.80(\mathrm{dt}, J=4.4,10.0 \mathrm{~Hz}, 1 \mathrm{H}), 2.70-$ $2.60(\mathrm{~m}, 1 \mathrm{H}), 2.50-2.34(\mathrm{~m}, 2 \mathrm{H}), 2.15-2.05(\mathrm{~m}, 1 \mathrm{H}), 1.85-1.52$ $(\mathrm{m}, 4 \mathrm{H}), 1.29-1.23(\mathrm{~m}, 1 \mathrm{H})$.

(S)-2-[(R)-1-(4-Methoxyphenyl)-2-nitroethyl]cyclohexanone (6c): ${ }^{9}$ Yield: $83 \%$; $[\alpha]_{\mathrm{D}}^{25}=-16.7\left(c=0.30\right.$ in $\left.\mathrm{CHCl}_{3}\right)$; HPLC analysis: $t_{\mathrm{R}}$ (minor) $=37.55 \mathrm{~min}, t_{\mathrm{R}}$ (major) $=49.98 \mathrm{~min}$, $93 \%$ ee. ${ }^{1} \mathrm{H}$ NMR $\left(300 \mathrm{MHz}, \mathrm{CDCl}_{3}\right) \delta 7.10$ (dd, $J=2.4,9.3$ $\mathrm{Hz}, 2 \mathrm{H}), 6.85-6.75$ (m, 2H), 4.93 (dd, $J=4.8,12.3 \mathrm{~Hz}, 1 \mathrm{H})$, 4.61 (dd, $J=9.9,12.3 \mathrm{~Hz}, 1 \mathrm{H}), 3.79(\mathrm{~s}, 3 \mathrm{H}), 3.72$ (dt, $J=4.5$, $9.9 \mathrm{~Hz}, 1 \mathrm{H}), 2.70-2.58(\mathrm{~m}, 1 \mathrm{H}), 2.55-2.35(\mathrm{~m}, 2 \mathrm{H}), 2.15-2.00$ $(\mathrm{m}, 1 \mathrm{H}), 1.85-1.49(\mathrm{~m}, 4 \mathrm{H}), 1.30-1.15(\mathrm{~m}, 1 \mathrm{H})$.

(S)-2-[(R)-1-(4-Bromophenyl)-2-nitroethyl]cyclohexanone (6d): ${ }^{11}$ Yield: $80 \%$; $[\alpha]_{\mathrm{D}}^{25}=-27.7\left(c=0.43\right.$ in $\left.\mathrm{CHCl}_{3}\right)$; HPLC analysis: $t_{\mathrm{R}}($ minor $)=27.76 \mathrm{~min}, t_{\mathrm{R}}($ major $)=32.84 \mathrm{~min}, 94 \%$ ee. ${ }^{1} \mathrm{H}$ NMR (300 MHz, $\left.\mathrm{CDCl}_{3}\right) \delta 7.46(\mathrm{~d}, J=8.7 \mathrm{~Hz}, 2 \mathrm{H}), 7.10$ $(\mathrm{d}, J=8.1 \mathrm{~Hz}, 2 \mathrm{H}), 4.95$ (dd, $J=4.5,12.6 \mathrm{~Hz}, 1 \mathrm{H}), 4.60$ (dd, $J=9.9,12.6 \mathrm{~Hz}, 1 \mathrm{H}), 3.77(\mathrm{dt}, J=4.5,9.9 \mathrm{~Hz}, 1 \mathrm{H}), 2.70-2.50$ $(\mathrm{m}, 1 \mathrm{H}), 2.48-2.30(\mathrm{~m}, 2 \mathrm{H}), 2.15-2.00(\mathrm{~m}, 1 \mathrm{H}), 1.90-1.49(\mathrm{~m}$, $4 \mathrm{H}), 1.32-1.10(\mathrm{~m}, 1 \mathrm{H})$.

(S)-2-[(S)-1-(Furan-2-yl)-2-nitroethyl] cyclohexanone (6e): ${ }^{11}$ Yield: $88 \%$; $[\alpha]_{\mathrm{D}}^{25}=-15.2\left(c=0.40\right.$ in $\left.\mathrm{CHCl}_{3}\right)$; HPLC analysis: $t_{\mathrm{R}}($ minor $)=32.12 \mathrm{~min}, t_{\mathrm{R}}$ (major) $=39.36 \mathrm{~min}, 90 \%$ ee. ${ }^{1} \mathrm{H}$ NMR $\left(300 \mathrm{MHz}, \mathrm{CDCl}_{3}\right) \delta 7.35(\mathrm{~m}, 1 \mathrm{H}), 6.30(\mathrm{dd}, J=1.8,3.3$ $\mathrm{Hz}, 1 \mathrm{H}), 6.15$ (d, J=3.3 Hz, 1H), 4.80-4.60 (m, 2H), 3.97 (dt, $J=4.8,9.3 \mathrm{~Hz}, 1 \mathrm{H}), 2.80-2.71(\mathrm{~m}, 1 \mathrm{H}), 2.50-2.25(\mathrm{~m}, 2 \mathrm{H})$, $2.25-2.00(\mathrm{~m}, 1 \mathrm{H}), 1.95-1.55(\mathrm{~m}, 4 \mathrm{H}), 1.40-1.23(\mathrm{~m}, 1 \mathrm{H})$.

(S)-2-((R)-1-(2-Bromophenyl)-2-nitroethyl)cyclohexanone (6f): ${ }^{11}$ Yield: $83 \%$; $[\alpha]_{\mathrm{D}}^{25}=-12.7\left(c=0.31\right.$ in $\left.\mathrm{CHCl}_{3}\right)$; HPLC analysis: $t_{\mathrm{R}}($ minor $)=28.37 \mathrm{~min}, t_{\mathrm{R}}($ major $)=34.70 \mathrm{~min}, 91 \%$ ee . ${ }^{1} \mathrm{H}$ NMR $\left(300 \mathrm{MHz}, \mathrm{CDCl}_{3}\right) \delta 7.49(\mathrm{dd}, J=1.2,8.1 \mathrm{~Hz}, 1 \mathrm{H})$, 7.25-7.15 (m, 2H), 7.08-7.00 (m, 1H), 4.85-4.70 (m, 2H), 4.30$4.20(\mathrm{~m}, 1 \mathrm{H}), 2.80(\mathrm{~m}, 1 \mathrm{H}), 2.35-2.25(\mathrm{~m}, 2 \mathrm{H}), 2.05-1.97(\mathrm{~m}$, $1 \mathrm{H}), 1.75-1.45(\mathrm{~m}, 4 \mathrm{H}), 1.30-1.26(\mathrm{~m}, 1 \mathrm{H})$.

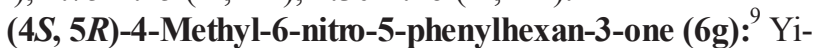
eld: $77 \% ;[\alpha]_{\mathrm{D}}^{20}=28.6\left(c=0.40\right.$ in $\left.\mathrm{CHCl}_{3}\right)$; HPLC analysis: $t_{\mathrm{R}}$ (minor) $=21.59 \mathrm{~min}, t_{\mathrm{R}}$ (major) $=26.64 \mathrm{~min}, 92 \%$ ee. ${ }^{1} \mathrm{H} \mathrm{NMR}$ $\left(300 \mathrm{MHz}, \mathrm{CDCl}_{3}\right) \delta$ 7.35-7.20 (m, 3H), 7.18-7.10 (m, 2H), 4.75-4.55 (m, 2H), $3.68(\mathrm{dt}, J=4.8,9.3 \mathrm{~Hz}, 1 \mathrm{H}), 3.05-2.95(\mathrm{~m}$, $1 \mathrm{H}), 2.70-2.55(\mathrm{~m}, 1 \mathrm{H}), 2.45-2.35(\mathrm{~m}, 1 \mathrm{H}), 1.06(\mathrm{t}, J=7.2 \mathrm{~Hz}$, $3 \mathrm{H}), 0.95(\mathrm{~d}, J=6.9 \mathrm{~Hz}, 3 \mathrm{H})$.

(R)-5-Nitro-4-phenylpentan-2-one (6h): ${ }^{3 \mathrm{~d}}$ Yield: 79\%; $[\alpha]_{\mathrm{D}}^{25}=$ $-22.8\left(c=0.51\right.$ in $\left.\mathrm{CHCl}_{3}\right)$; HPLC analysis: $t_{\mathrm{R}}$ (minor) $=41.19$ $\min , t_{\mathrm{R}}($ major $)=47.07 \mathrm{~min}, 86 \%$ ee. ${ }^{1} \mathrm{HNMR}\left(300 \mathrm{MHz}, \mathrm{CDCl}_{3}\right)$ $\delta 7.40-7.20(\mathrm{~m}, 5 \mathrm{H}), 4.71(\mathrm{dd}, J=6.9,12.3 \mathrm{~Hz}, 1 \mathrm{H}), 4.62$ (dd, $J=7.5,12.3 \mathrm{~Hz}, 1 \mathrm{H}), 4.05-3.95(\mathrm{~m}, 1 \mathrm{H}), 2.91(\mathrm{~d}, J=6.9 \mathrm{~Hz}$, $2 \mathrm{H}), 2.15$ (s, 3H).

(S)-2-[(R)-2-Nitro-1-phenylethyl] cyclopentanone (6i): ${ }^{11} \mathrm{Yi}-$ eld: $71 \% ;[\alpha]_{\mathrm{D}}^{25}=-19.7\left(c=0.60\right.$ in $\left.\mathrm{CHCl}_{3}\right)$; HPLC analysis: $t_{\mathrm{R}}$ $($ minor $)=35.24 \mathrm{~min}, t_{\mathrm{R}}$ (major) $=42.28 \mathrm{~min}, 88 \%$ ee. ${ }^{1} \mathrm{H} \mathrm{NMR}$ $\left(300 \mathrm{MHz}, \mathrm{CDCl}_{3}\right) \delta 7.40-7.25(\mathrm{~m}, 3 \mathrm{H}), 7.25-7.11(\mathrm{~m}, 2 \mathrm{H}), 5.33$ $(\mathrm{dd}, J=5.7,12.9 \mathrm{~Hz}, 1 \mathrm{H}), 4.71(\mathrm{dd}, J=10.2,12.6 \mathrm{~Hz}, 1 \mathrm{H}), 3.75-$ $3.64(\mathrm{~m}, 1 \mathrm{H}), 2.45-2.30(\mathrm{~m}, 2 \mathrm{H}), 2.25-2.05(\mathrm{~m}, 1 \mathrm{H}), 2.00-1.80$ (m, 2H), 1.78-1.55 (m, 1H), 1.53-1.35 (m, 1H).

(R)-6-Nitro-5-phenylhexan-3-one (6j): ${ }^{11}$ Yield: $72 \%$; $[\alpha]_{\mathrm{D}}^{25}=$ $-10.12\left(c=0.35\right.$ in $\left.\mathrm{CHCl}_{3}\right)$; HPLC analysis: $t_{\mathrm{R}}$ (minor) $=17.37$ $\min , t_{\mathrm{R}}($ major $)=24.15 \mathrm{~min}, 85 \%$ ee. ${ }^{1} \mathrm{HNMR}\left(300 \mathrm{MHz}, \mathrm{CDCl}_{3}\right)$ $\delta$ 7.35-7.25 (m, 3H), 7.24-7.20 (m, 2H), 4.75-4.54 (m, 2H), 4.08-3.99 (m, 1H), 2.89 (d, J = 7.2 Hz, 2H), 2.45-2.31 (m, 2H), $1.02(\mathrm{t}, \mathrm{J}=7.2 \mathrm{~Hz}, 3 \mathrm{H})$.

\section{Conclusions}

In conclusion, we have synthesized a new chiral catalyst 2. it was found that catalyst $\mathbf{2}$ could catalyzed the asymmetric Michael reaction of ketones with nitroolefins smoothly at room temperature, giving the desired adduct $7 \mathbf{a}-\mathbf{j}$ in $71-92 \%$ yields with excellent diastereoselectivities (syn/anti $=90 / 10-97 / 3)$ and enantioselectivities (up to $95 \%$ ee).

\section{References}

1. Berner, O. M.; Tedeschi, L.; Enders, D. Eur. J. Org. Chem. 2002, 1877.

2. For example: (a) Luchaco-Cullis, C. A.; Hoveyda, A. H. J. Am. Chem. Soc. 2002, 124, 8192. (b) Duursma, A.; Minnaard, A. J.; Feringa, B. L. J. Am. Chem. Soc. 2003, 125, 3700.

3. Some papers after 2006: (a) Brandau, S.; Landa, A.; Franzen, J.; Marigo, M.; Jørgensen, K. A. Angew. Chem., Int. Ed. 2006, 45, 4305. (b) Bartoli, G.; Bosco, M.; Carlone, A.; Cavalli, A.; Locatelli, M.; Mazzanti, A.; Ricci, P.; Sambri, L.; Melchiorre, P. Angew. Chem., Int. Ed. 2006, 45, 4966. (c) Wang, Y.; Liu, X. F.; Deng, L. J. Am. Chem. Soc. 2006, 128, 1928. (d) Huang, H. B.; Jacobsen, E. N. J. Am. Chem. Soc. 2006, 128, 7170. (e) Diner, P.; Nielsen, M.; Marigo, M.; Jørgensen, K. A. Angew. Chem., Int. Ed. 2007, 46, 1983. (f) Tang, B.; Zeng, X. F.; Lu, Y. P.; Chua, P. J.; Zhong, G. F. Org. Lett. 2009, 11, 1927. (g) Lo, C. M.; Chow, H. F. J. Org. Chem. 2009, 74, 5181. (h) Zeng, X. F.; Zhong, G. F. Synthesis 2009, 1545. (i) Shah, J.; Khah, S. S.; Blumenthal, H.; Liebscher, J. Synthesis 2009, 3975.

4. For example: (a) Okino, T.; Hoashi, Y.; Takemoto, Y. J. Am. Chem. Soc. 2003, 125, 12672. (b) Okino, T.; Hoashi, Y.; Furukawa, T.; Xu, X. N.; Takemoto, Y. J. Am. Chem. Soc. 2005, 127, 119. (c) Hayashi, Y.; Okino, T.; Takemoto, Y. Angew. Chem., Int. Ed. 2005, 44, 4032. (d) Tsogoeva, S. B.; Wei, S. Chem. Commun. 2006, 1451. (e) Doyle, A. G.; Jacobsen, E. N. Chem. Rev. 2007, 107, 5713.

5. For example: (a) Hoashi, Y.; Okino, T.; Takemoto, Y. Angew. Chem. Int. Ed. 2005, 44, 4032. (b) Tsogoeva, S. B.; Wei, S. W. Chem. Commun. 2006, 1451. (c) Okino, T.; Hoashi, Y.; Furukawa, T.; Wu, X. N.; Takemoto, Y. J. Am. Chem. Soc. 2005, 127, 119. (d) Cao, C. L.; Sun, X. L.; Zhou, J. L.; Tang, Y. J. Org. Chem. 2007, 72, 4073. (e) Cao, C. L.; Ye, M. C.; Sun, X. L.; Tang, Y. Org. Lett. 2006, 8, 2901. (f) Jiang, X. X.; Zhang, Y. F.; Chan, A. S. C.; Wang, R. Org. Lett. 2009, 11, 153. (g) Cao, Y. J.; Lu, H. H.; Lai, Y. Y.; Lu, L. Q.; Xiao, W. J. Synthesis 2006, 3795. (h) Cheng, D. P.; Bao, W. L. Lett. Org. Chem. 2008, 5, 342. (i) Mandal, T.; Zhao, C. G. Angew. Chem., Int. Ed. 2008, 47, 7714. (j) Lu, A. D.; Gao, P.; Wu, Y.; Wang, Y. M.; Zhou, Z. H.; Tang, C. C. Org. Biomol. Chem. 2009, 7, 3141 .

6. Dahlin, N.; Boegevig, A.; Adolfsson, H. Adv. Synth. Catal. 2004, 346, 1101.

7. (a) Kando, Y.; Uneme, H.; Minamida, I. Eur. Pat. Appl. 1991 EP 452782. (b) Wakita, T.; Kinoshita, K.; Yasui, N.; Yamada, E.; Kawahara, N.; Kodaka, K. J. Pestic. Sci. 2004, 29, 348.

8. Kumaran, G.; Kulkarni, G. H. Synthesis 1995, 1545.

9. Wang, J.; Li, H.; Lou, B.; Zu, L. S.; Guo, H.; Wang, W. Chem. Eur. J. 2006, 12, 4321.

10. Tan, B.; Zeng, X. F.; Lu, Y. P; Chua, P. J.; Zhong, G. F. Org. Lett. 2009, 11, 1927.

11. Cao, C. L.; Ye, M. C.; Sun, X. L.; Tang, Y. Org. Lett. 2006, 8, 2904. 